in the Wales Deanery (UK). Grounded theory was used to analyse the responses, including free text.

While $8 \%$ of respondents cited lack of knowledge of or disagreement with the practise, $87 \%$ of obstetricians and trainees admitted that as the surgical sequence involved in a delivery is automatic, they sometimes or often forgot to incorporate a delay before clamping. This automaticity is adaptive, arising from a need to reduce cognitive load during complex motor tasks.

Where visuospatial skills are sufficiently refined (task mastery), cognitive attentional skills are engaged only at key points. These are used as landmarks in expert sequences such as playing a musical instrument, using a gaming controller or surgery. During an abdominal delivery, clamping of the cord is unlikely to be a key landmark. This applies particularly for the experienced obstetrician in contrast to the novice.

The hypothesis that there would be an inverse relationship between the experience of the surgeon and the ease of incorporation of a new element to the operation was supported. Simple aide memoires facilitated incorporation of the new process, shown at reaudit.

\section{PL.66 VAGINAL BREECH DELIVERY - 12 YEARS AFTER THE TERM BREECH TRIAL ARE THE RISKS AS HIGH AS SUGGESTED? AUDIT OF PRACTISE WITHIN THE SETTING OF A HIGH RISK LABOUR WARD}

doi:10.1136/archdischild-2013-303966.248

C Mullan, N Musial, L Byrd. St. Mary's Hospital, Manchester, UK

The publication of the Term Breech Trial 12 years ago caused an almost universal change in UK practise towards offering elective caesarean section to women with breech presentation at term. These results have entered the public psyche to such an extent that women no longer have confidence undergoing vaginal breech deliveries (VBD) and experience has been eroded such that clinicians no longer maintain their skills. This has implications for women wishing to undergo $\mathrm{VBD}$ as well as morbidity and mortality from increasing numbers of caesarean sections (CS). We sought to answer the question of whether it was suitable and safe to offer VBD to women presenting in labour rather than carrying out emergency CS.

Between 2002-2011, 502 women with breech presentation at term attended in labour. 77 (15\%) had normal vaginal delivery, 219 (44\%) had emergency CS and 206 (41\%) had urgent CS. In the CS group, 51 (12\%) required CS anyway; therefore $78 \%$ were suitable to consider VBD.

Only $47 \%$ of this group attempted VBD. The majority chose CS due to personal preference or recommendation from doctors, highlighting lack of confidence in VBD by both doctors and women.

5-minute Apgar scores were comparable between groups but morbidity from blood loss was higher in women undergoing emergency CS vs. VBD. (1250 ml vs. $686 \mathrm{ml})$.

We believe these results show that VBD can be safe in selected cases and the time has come for clinicians to regain their experience so that more women can be offered this birth option with confidence.

\section{PL.67 EFFECT OF DIFFERENT PROSTAGLANDIN PREPARATIONS FOR INDUCTION ON ADMISSION TO DELIVERY TIME}

doi:10.1136/archdischild-2013-303966.249

${ }^{2} \mathrm{~A}$ Rahim-Babar, 'S Bonner, ${ }^{1} \mathrm{C}$ Tower. ${ }^{1}$ Central Manchester University Hospitals NHS Foundation Trust, Manchester, UK; ${ }^{2}$ University of Manchester, Manchester, UK

Background Around 20\% of labours are induced with the current recommended method being prostaglandin for cervical priming. The 2 commonly used preparations are a prostaglandin gel administered 6 hourly, or a prostaglandin pessary, administered over 24 hours. Following a change in protocol from gel to pessary, we aimed to look at the effect on admission to delivery time (ADT).
Methods The notes of 395 women who had labour induced within a large tertiary unit in the North West between August 2009 and May 2012 were studied, excluding those induced for prelabour ruptured membranes. Data was collected retrospectively using a standard proforma.

Results 93 women received prostaglandin gel and 302 received the pessary (28 also needed gel). The median ADT for women induced using gel was significantly shorter at 26.37 hours (interquartile range 15.87-42.96) than with the pessary at 31.83 hours (20.7346.54 hours $p=0.002$ non-parametric testing). There was no difference in parity or oxytocin use between the 2 groups. Outcomes between the 2 groups were the same, with no difference in postpartum haemorrhage rate or vaginal delivery $(p>0.05)$.

Conclusions The prostaglandin pessary was associated with a longer $\mathrm{ADT}$, which is perhaps unsurprising given its longer duration of use prior to assessment for amniotomy. This is probably because the predicted increase in labour commencing in the pessary group without further oxytocin did not occur, reflected in no difference in oxytocin use between the 2 groups. This has implications for bed occupancy, patient flows and NHS costs.

\section{PL.68 WHEN SHOULD TWIN INDUCTION OF LABOUR START TO INCREASE THE CHANCES OF DAYTIME DELIVERY?}

doi:10.1136/archdischild-2013-303966.250

${ }^{1} \mathrm{M}$ Tattersall, ${ }^{2} \mathrm{~L}$ Bricker. 'University of Liverpool, Liverpool, UK; '2Liverpool Women's Hospital, Liverpool, UK

Background Vaginal twin deliveries can be complicated and senior obstetrician presence may be advisable. Our unit has consultant on-site presence between 08:30 and 20:30, so we sought to determine when inductions should be commenced to maximise deliveries during these "daytime" hours.

Methods Women having prostaglandin induction after 36 weeks, resulting in at least one vaginal delivery and where no delays to normal care occurred were selected. Nulliparous and parous women were considered separately and the percentage of "daytime" deliveries calculated for inductions commenced in the morning (06:00 11:59), afternoon (12:00 - 17:59) and evening (18:00 - 23:59). (Inductions commenced 00:00 - 05:59 were excluded due to infrequency.) Analysis of length of labour (defined from start time to delivery of first twin) was performed.

Results The majority of inductions were commenced in the morning. For nulliparous women, $71 \%$ of morning-commenced inductions resulted in "daytime" deliveries, compared with $50 \%$ and $67 \%$ of afternoon-commenced and evening-commenced procedures. Labour length was normally distributed with mean of 21.8 hours (SD 7.9 hours). For parous women, afternoon-commenced induction produced a higher percentage of "daytime" deliveries; $85 \%$ compared with $50 \%$ and $67 \%$ for morning-commenced and eveningcommenced inductions. Labour length was normally distributed with mean of 15.0 hours (SD 7.0 hours).

Conclusions For nulliparous women, commencing induction in the morning provides a high likelihood of "daytime" delivery. For parous women, analysis of inductions by start time and mean length of labour suggests a trial of commencing induction later in the day might increase the proportion of "daytime" deliveries.

\section{PL.69 DELIVERY OUTCOMES AND EVENTS IN SUBSEOUENT PREGNANCIES AFTER PREVIOUS ANAL SPHINCTER INJURY}

doi:10.1136/archdischild-2013-303966.251

AA Ali, KG Glennon, SY Yousif, ME Eogan. Rotunda Hospital, Dublin, Ireland

Introduction Few recent studies have examined outcome in pregnancies following previous third degree perineal tears and results are 